\title{
Rehabilitation of patients with venous diseases of the lower limbs: State of the art
}

(C) The Author(s) 2018

Reprints and permissions: sagepub.co.uk/journalsPermissions.nav DOI: I0.II77/02683555/8754463 journals.sagepub.com/home/phl SAGE

\author{
Alberto Caggiati', Marianne De Maeseneer², Attilio Cavezzi ${ }^{3}$, \\ Giovanni Mosti ${ }^{4}$ and Nick Morrison ${ }^{5}$
}

\begin{abstract}
Background: To date, no document comprehensively focused on the complex issue of the rehabilitation of chronic venous diseases of the lower limbs.

Method: This article overviews and summarizes current strategies concerning venous rehabilitation of lower limbs.

Results: Venous rehabilitation is based on four main strategies: (I) lifestyle adaptations and occupational therapies; (2) physical therapies; (3) adapted physical activities; (4) psychological and social support. Rehabilitative protocols must be tailored to the specific needs of each patient, depending on the severity of chronic venous disease and on the location and pattern of venous lesion(s), but also on age, motor deficits, co-morbidities and psychosocial conditions.

Conclusions: Venous rehabilitation consists of non-pharmacologic and non-surgical interventions aiming at prevention of venous disease progression and complications, reduction of symptoms and improvement of quality of life. Welldesigned clinical trials are required to evaluate the efficacy of the described rehabilitative protocols in influencing the evolution of venous disorders.
\end{abstract}

\section{Keywords}

Venous diseases, post-thrombotic syndrome, chronic venous insufficiency, chronic venous disease, calf muscle pump function

\section{Introduction}

Rehabilitation of patients with venous diseases of the lower limbs consists of all non-pharmacologic and nonsurgical interventions aiming at prevention of venous disease progression and complications, reduction of symptoms, and improvement of quality of life $(\mathrm{QoL}){ }^{1}$

With the exception of medical elastic compression stockings (MECS), physical therapies (PTs), and rehabilitative protocols are not or only partially mentioned in recent guidelines on chronic venous diseases (CVDs). ${ }^{2}$ In turn, there is increasing evidence regarding the role of PTs in preventing disease progression and optimizing results of surgical and pharmacologic treatments.

This study aims to overview and summarize current strategies concerning rehabilitation of patients with venous diseases of the lower limbs.

\section{Methods}

To start the present study, a literature search was initially designed and conducted by A.C. in PubMed,
Embase, and the Cochrane Library between 1 January 1980 and 1 July 2017. Criteria for search and selection were the following headings and keywords: Rehabilitation, Physical Therapy, Adapted Physical Activities, Sport, Hydrotherapy, Balneotherapy, Salus Per Aquam (SPA) and Venous Disease, Venous Insufficiency, Venous Ulcer, Varicose Veins, PostThrombotic Syndrome. By reference checking and additional search by the Authors, other relevant literature was added.

\footnotetext{
'Department of Anatomy, Sapienza University, Rome, Italy

${ }^{2}$ Department of Dermatology, Erasmus MC, Rotterdam, The Netherlands

${ }^{3}$ Stella Maris Hospital, San Benedetto del Tronto, Italy

${ }^{4}$ Barbantini Hospital, Lucca, Italy

${ }^{5}$ Morrison Vein Institute, Tempe, AZ, USA

Corresponding author:

Alberto Caggiati, Department of Anatomy, Sapienza University, Via A. Borelli 50, Rome, Italy.

Email: Alberto.caggiati@uniromal.it
} 
Unfortunately, studies available in literature largely focus only on patients with severe chronic venous insufficiency (CVI) (C5 and C6 legs, according to CEAP classification). Moreover, most studies are not corroborated by evidence-based analysis of results. For these reasons, all advices in the present document should be currently considered as "best practice suggestions."

\section{The need for a tailored rehabilitation approach}

The rehabilitation approach must be tailored to the specific needs of each patient. In fact, it not only depends on the severity of CVD and on location and pattern of venous lesions but also on age, motor deficits, co-morbidities, and psychosocial conditions.

It is paramount to consider all co-morbidities potentially influencing venous return and to treat them whenever possible (obesity, cardiac or respiratory insufficiency, renal failure and metabolic disorders, neurologic, muscular or articular diseases and finally, cutaneous lesions). In particular, rehabilitative protocols must be designed taking into account the cardiorespiratory, skeletal, neuromuscular, and psychosocial limitations of each patient.

Obesity is the main comorbidity to be considered in patients with CVI. ${ }^{3}$ In fact, literature data show that CEAP clinical class is more advanced in obese than in non-obese patients with comparable severity of venous incompetence. ${ }^{3}$

\section{Tools for rehabilitation}

Rehabilitation of patients with CVD of the lower limbs is based on four main strategies: (1) lifestyle adaptations and occupational therapies; (2) PTs; (3) adapted physical activities (APAs); (4) psychosocial support (Table 1).

\section{Lifestyle adaptations}

All CVI patients should adapt their lifestyle according to the clinical severity of the disease.

The most frequent advice is to start wearing MECS during day time. The efficacy of MECS in chronic phases of venous diseases is unclear, due to the low quality of most of the studies. ${ }^{2}$ The role of MECS in the prevention of post-thrombotic syndrome (PTS) is still debated. ${ }^{4,5}$ Nevertheless, MECS improve patients' symptoms ${ }^{6}$ and QoL. ${ }^{7}$ Moreover, there is a large body of evidence on the positive effect of MECS in promoting ulcer healing ${ }^{8}$ and preventing ulcer recurrence. ${ }^{9}$

In addition to MECS, other lifestyle changes may be advised, as listed in Table 2. These suggestions aim to improve the QoL by reducing venous stasis and related symptoms, but also possibly preventing complications and delaying disease progression. ${ }^{10}$ Even if only some of these advices are supported by evidence-based studies, ${ }^{11}$ lifestyle changes and self-care strategies should be considered in all patients with venous disorders.

Table I. Main strategies for the rehabilitation of patients with venous diseases.

\begin{tabular}{|c|c|c|c|}
\hline Strategy & Recommended in & Objectives & Main tools \\
\hline $\begin{array}{l}\text { Lifestyle } \\
\text { adaptations }\end{array}$ & All patients with venous disease & $\begin{array}{l}\text { To reduce symptoms and improve the } \\
\text { QoL.To possibly prevent complica- } \\
\text { tions, disease progression and } \\
\text { recurrence }\end{array}$ & $\begin{array}{l}\text { MECS } \\
\text { Advices mentioned in Table } 2\end{array}$ \\
\hline PTs & $\begin{array}{l}\text { All patients showing impairment } \\
\text { of the mechanisms facilitating } \\
\text { venous return. } \\
\text { All patients with comorbidities } \\
\text { which negatively influence } \\
\text { venous return }\end{array}$ & $\begin{array}{l}\text { To obtain a persistent increase in } \\
\text { efficacy of the mechanisms which } \\
\text { facilitate venous return }\end{array}$ & $\begin{array}{l}\text { Plantar correction } \\
\text { Improve muscular efficiency } \\
\text { Improve joints flexibility } \\
\text { Gait reeducation } \\
\text { Respiratory exercises } \\
\text { Exercise mimetics } \\
\text { Treatment of comorbidities }\end{array}$ \\
\hline $\begin{array}{l}\text { APAs and } \\
\text { recreational } \\
\text { sports }\end{array}$ & $\begin{array}{l}\text { Poorly active patients, obese } \\
\text { patients }\end{array}$ & $\begin{array}{l}\text { To maintain and possibly improve the } \\
\text { results of PT } \\
\text { To possibly prevent complications, dis- } \\
\text { ease progression and recurrence }\end{array}$ & $\begin{array}{l}\text { Supervised physical activity tele- } \\
\text { rehabilitation (home activity) } \\
\text { Hydrotherapy and SPA therapies }\end{array}$ \\
\hline $\begin{array}{l}\text { Psychological } \\
\text { and/or social } \\
\text { support }\end{array}$ & $\begin{array}{l}\text { Patients with psychic and/or } \\
\text { social problems }\end{array}$ & $\begin{array}{l}\text { To obtain a better adherence to } \\
\text { rehabilitative protocols (PT, APAs, } \\
\text { elastic stockings, lifestyle advice) } \\
\text { To improve the QoL }\end{array}$ & $\begin{array}{l}\text { Increased measures of social } \\
\text { interaction and support; } \\
\text { psychotherapy }\end{array}$ \\
\hline
\end{tabular}

QoL: quality of life; MECS: medical elastic compression stockings; PTs: physical therapies; APAs: adapted physical activities; SPA: Salus Per Aquam. 
Table 2. Main lifestyle changes and self-care for patients with venous disorders of the lower limbs.

- To walk frequently, especially during working days, preferably in the middle of the day or before/after work while always wearing MECS

- To rest with the legs elevated during breaks and whenever possible

- To raise the foot end of the bed $10-20 \mathrm{~cm}$ with blocks to obtain an angle of approximately $10^{\circ}$. This is not recommended for patients with cardiac or pulmonary failure, peripheral arterial disease and hiatal hernia

- To maintain skin moisture and prevent drying out and cracking of the skin

- To possibly use specially designed seats at work

- To take cold showers to counteract vasodilatation, especially in the warm season

- To wear appropriate clothing: loose clothes avoiding constrictions and favoring respiratory movements are to be preferred

- To wear comfortable shoes with low heels $(<3 \mathrm{~cm})$, supplied by insoles in case of foot dysmorphisms

- To avoid wearing high heels

- To avoid sunbathing

- To avoid sauna, hot thermal baths and mud baths

MECS: medical elastic compression stockings.

Table 3. The vis a latere: main venous pumps of the lower limb.

\begin{tabular}{|c|c|c|c|}
\hline Site of pumping & Muscles & Movement & Compressed veins \\
\hline Foot & Plantar muscles & Weight-bearing & Lateral plantar veins \\
\hline Leg (lower half) & Anterior muscles & Dorsiflexion of the foot & $\begin{array}{l}\text { Deep veins at the ankle } \\
\text { and lower leg }\end{array}$ \\
\hline Leg (anterior compartment) & Anterior muscles & Dorsiflexion of the foot & $\begin{array}{l}\text { Deep veins of the antero-lateral } \\
\text { compartment }\end{array}$ \\
\hline Leg (posterior compartment) & Sural muscles & Plantar flexion of the foot & Sural veins \\
\hline Popliteal fossa & $\begin{array}{l}\text { Heads of the } \\
\text { gastrocnemius }\end{array}$ & Contraction of the gastrocnemius & Popliteal vein (lower portion) \\
\hline \multirow[t]{2}{*}{ Anterior thigh } & Sartorius & Flexion of the hip/extension of the knee & Femoral vein \\
\hline & Quadriceps & Flexion of the hip/extension of the knee & Femoral vein \\
\hline Posterior thigh & Hamstring & Flexion of the knee & Deep femoral vein \\
\hline Buttock & Gluteal muscles & Extension of the hip & Gluteal veins \\
\hline
\end{tabular}

Note: Modified from Gardner and Fox. The return of blood to the heart. ${ }^{16}$

\section{PTs in CVI patients}

The aim of PTs is to obtain a persistent increase in the efficacy of the mechanisms facilitating venous return. Patients with severe CVI (C4-C6 legs, according to the CEAP classifications) or comorbidities influencing venous function, need to be considered for PTs.

The blood return from the lower limbs is guaranteed by three kinds of forces: (1) the vis a tergo (blood propulsion by residual capillary pressure), (2) the vis a latere (blood acceleration by muscular pumping), and finally, (3) the vis a fronte (thoraco-abdominal blood aspiration). PTs aim to increase the efficacy of all mechanisms involved in blood acceleration and aspiration.

Increasing the vis a tergo. The vis a tergo consists of the residual pressure of the blood at the end of the capillary bed. It was demonstrated that exercising enhances microvascular endothelial function, resulting in increased venous flow, ${ }^{12}$ even if exercises are performed with other parts of the body. ${ }^{13,14}$

Accordingly, in order to increase the blood propulsion at the venular level, any kind of physical activity should be intensified and sedentary habits completely discouraged.

Increasing the vis a latere. The vis a latere consists of the blood acceleration due to the muscular contractions which squeeze intermuscular veins and intramuscular venous networks (muscular pumps). A coordinated chain of muscular pumps is sequentially activated during walking, respectively, the plantar, calf, thigh and gluteal pumps (Table 3). ${ }^{15,16}$

In patients with severe CVI (C4-C6 classes), plantar loading, joint flexibility, and muscular efficiency are more impaired than in age-matched subjects. Hence, to increase the vis a latere, it may be necessary to 
correct plantar abnormalities, to increase muscular efficiency, and joint mobility, as well as to optimize function and coordination of the different pumps.

The plantar pump. The role of the plantar pump in venous return is well established. ${ }^{17,18}$ It has been demonstrated that the hemodynamic effects of the plantar pump are quantitatively similar to those of the calf ${ }^{17}$ and that correction of plantar loading by insoles improves venous return and QoL with an efficiency that is almost equal to that provided by MECS. ${ }^{19}$ For all these reasons, the plantar loading must be routinely evaluated in all patients with CVI and possibly corrected.

Ankle rehabilitation. Mobility of the ankle joints is reduced in patients with CVI, in a greater proportion than in similarly aged subjects without venous disorders. ${ }^{20-22}$ The reduction of the ankle range-of-motion (aROM) correlates to the severity of the venous disease. ${ }^{20}$ In limbs classified $\mathrm{C} 4$ and higher, this can be due to periostitis, calcification, and/or lipodermatosclerosis with subcutaneous and fascial fibrosis. ${ }^{23}$

PT for ankle rehabilitation consists of passive and active movements of the ankle starting with nonweight-bearing exercises, moving to resistive exercises, and then weight-bearing activities. ${ }^{24}$ Apart from these traditional physiotherapeutic treatments, ankle flexibility can also be enhanced by manual lymphatic drainage, ${ }^{23}$ biomechanical stimulations, ${ }^{25}$ and myofascial release. ${ }^{26}$ Increasing a ROM correlates to improvement of the calf pump efficiency. ${ }^{27}$

The calf pump. The hemodynamic role of the calf pump in venous return is well established. In legs with severe venous disease, the calf pump is often dysfunctional and can lead to ulcer formation, delayed ulcer healing, and increased risk of ulcer recurrence. ${ }^{26}$

Action of the calf pump: The mechanism of action of the calf pump is the alternate contraction of the extensor and flexor muscles of the leg during the gait. The contraction of the extensor muscles squeezes the deep veins of the lower leg (distal leg pump ${ }^{16}$ ) and of the anterolateral leg. The contraction of the flexor muscles squeezes the sinusoids of the sural veins and the deep veins of the posterior leg (upper leg pump ${ }^{16}$ ).

The efficacy of the calf pump depends on correct plantar loading, normal ankle mobility and, finally, muscular efficiency. Accordingly, besides static foot disorders and other skeletal anomalies, calf pump dysfunction may be related to abnormalities of muscle strength and endurance. Impaired muscular efficiency is due to age, disuse, and venous incompetence itself. In fact, the latter was recognized to possibly cause muscle atrophy ${ }^{28,29}$ and peripheral neuropathy. ${ }^{30}$
Improving calf pump function: It has been clearly demonstrated that calf exercise regimens improve muscular endurance ${ }^{31}$ and may even restore proper function of the muscle pump with increased ejection fraction $(E F)$ and reduced residual fraction $(\mathrm{RF}){ }^{31}$ Rehabilitation of the calf pump mainly consists of exercises of dorsal and plantar foot flexion with increasing load. These can be performed according to different protocols. Individually tailored physio-therapeutic exercises may include a mix of stretching the muscles and repeated contractions and relaxations. Besides improving $\mathrm{EF}$ and diminishing $\mathrm{RF}$, these exercises also improve aROM.

Alternatively, calf pump efficiency can also be improved by exercise mimetics:

Foot movers: are specially designed training machines by which patients can exercise in a seated or a reclining position. The use of foot exercisers is included in most rehabilitation programs. ${ }^{32,33}$ However, no study evaluated the long-term adherence to the use of foot exercisers.

Artificial muscle activation: Rhythmic muscle activity can be achieved by transcutaneous muscle ${ }^{34}$ or nerve stimulation. ${ }^{35}$ The efficacy of these devices has been demonstrated to increase venous flow and prevent venous stasis, ${ }^{36}$ especially if used in conjunction with MECS. ${ }^{37}$

Intermittent pneumatic compression (IPC) was demonstrated to prevent deep vein thrombosis in subjects at risk, as well as to increase ulcer healing. ${ }^{38}$ No data are available concerning the effectiveness of IPC in chronic phases of venous diseases. As an example, no benefit was seen to accrue if IPC was used as an adjuvant therapy to aid prevention of ulcer recurrence. ${ }^{39}$ However, it was recently proposed to use intermittent compression devices or pneumatic compression sleeve units in patients with severe PTS whose symptoms are inadequately addressed with MECS alone. ${ }^{2}$

Gait re-education. A disturbed gait is typical for patients with severe CVI, especially for older ones. ${ }^{40}$ Apart from other neuromuscular dysfunctions typical for patients with venous diseases of the lower limbs, ${ }^{28-30}$ a deficit in proprioception may be responsible for gait alterations. ${ }^{41}$ Gait re-education may significantly increase the efficacy of the plantar and leg pumps ${ }^{39,41}$ by promoting heel-to-toe gait pattern and discouraging the shuffling gait.

The goal of gait re-education is to regain the correct sequence of weight bearing on the calcaneus (emptying of the veins of the lower leg and of the anterior muscular compartment), weight bearing on the mid-foot 
(emptying the plantar reservoir), and weight bearing on the anterior foot (emptying the veins of the calf).

The gait must be evaluated in all patients with venous disorders, and re-education can be prescribed in those showing abnormality of the physiologic sequence of foot, calf, and thigh motions during walking. $^{40}$

Once expelled from the leg, the blood is further propelled by the popliteal, thigh (anterior and posterior), and gluteal pumps ${ }^{17}$ (Table 3). Very few studies on venous rehabilitation considered the role of these pumps. Gait re-education contributes to improving the efficiency of these pumps and coordinating their sequential activation.

Increasing the vis a fronte. The vis a fronte consists of the thoraco-abdominal blood aspiration from the lower limbs. Valsalva described in 1710 the blood aspiration activated by respiratory variations of intra-thoracic pressure. Franklin and Janker ${ }^{42}$ described the blood aspiration from the inferior vena cava due to variation of the intrabdominal pressure as well as by spinal movements related to spine flexion-extension and rotation. Even if thoracoabdominal aspiration has been considered predominant over lower extremity muscle pumping in venous return, ${ }^{43}$ few protocols on venous rehabilitation include the necessity of specific respiratory exercises.

Development and implementation of respiratory exercise protocols for improving the venous return should therefore be further investigated.

\section{APAs}

Patients with severe CVI are less mobile and physically active than age-matched counterparts and only about $30 \%$ of them perform exercises regularly. ${ }^{44}$ In all these cases, programs of supervised exercises efficiently supplement daily physical activities. ${ }^{45}$

APAs can also be indicated in patients who received PT to maintain (and possibly improve) the results of the treatment and to prevent recurrences.

The goal of APAs is to increase the strength of muscles and the mobility of joints involved in the above described pumping mechanisms. APAs consist of physical or sports activities adapted to the capabilities of each patient, his/her preference, and potential co-morbidities. APA protocols are currently based on daily intensification of any kind of physical activity, structured aerobic exercises, structured resistance exercises, and flexibility exercises.

APAs can be performed in different settings, depending upon clinical severity and co-morbidities. Outpatients may follow rehabilitative APAs under the supervision of caregivers in the hospital setting or outside the hospital. APAs may be performed also at home. ${ }^{45}$ In the latter case, online coaching and remotecontrol instruments (tele-rehabilitation) are now available to promote the adherence to therapeutic protocols. $^{45}$

Short- and long-term APA programs of rehabilitation (from one week to six months) demonstrated positive results in terms of calf muscle pump function and reduction of symptoms. ${ }^{46}$

However, to date, no study established the effectiveness in preventing worsening and complications of CVII. ${ }^{46,47}$ Moreover, the adherence to physical activity programs is rather limited; in patients with venous ulceration, it appeared to be only about $40 \% .{ }^{44}$

Sports activities in patients with CVI. No evidence-based studies have been carried out on the positive or negative effects of sports in patients with CVI. Only anecdotal contributions are available. ${ }^{10}$ Appropriate sports are those which associate rhythmic movements of the muscles and joints of the lower limbs and deep breathing: walking, running, swimming, water aerobics, crosscountry skiing (in contrast to downhill skiing), golf, and dancing. ${ }^{10}$

Inappropriate sports activities are those which involve increasing the intra-abdominal pressure (weightlifting), blockage of the ankle joint (downhill skiing), and rapid increase of the pressure of the blood column on venous valves (tennis, ball games). Violent sports are discouraged as well, due to the risks for leg trauma. ${ }^{10}$

Physicians often encourage sedentary patients to train regularly and even participate in sports competitions. However, an excessive practice of some sports (e.g. long-distance running and cycling) may be inappropriate in patients with venous diseases, if not supported with proper MECS. In addition, exaggerated sports activity might even induce varicose changes or enhance pre-existing ones. ${ }^{48}$

It is still debated if "elastic sports stockings" are effective in increasing sports performance in healthy individuals. It seems that elastic stockings have a positive effect on muscle recovery associated to a greater comfort. ${ }^{49}$ Even if no study has scientifically evaluated the protective role of elastic stockings in athletes with venous diseases of the lower limbs, the use of elastic stockings by venous patients also during sports activities may be included in lifestyle suggestions.

Hydrotherapy and SPA therapies. Hydrotherapy has a long tradition in Europe for the treatment of vein diseases. ${ }^{50}$ Hydromassotherapy and vascular pathways are associated with immersion in sulfurous, sulfate, salsojoidic, salsobromojodic waters. Specific exercises have been 
designed to be performed in the water by patients with venous diseases of the lower limbs. ${ }^{51}$

The positive effects are related to specific properties of immersion in water due to hydrostatic pressure, osmotic pressure, and temperature (balneotherapy) and partly related to specific chemical properties of the water (crenotherapy). ${ }^{50,52-54}$

The efficacy of hydrotherapy is based almost purely on empiricism. No study has scientifically demonstrated the biological effects of particular components of water on venous return.

SPA sessions are usually performed in thermal resorts which allow a comprehensive approach to venous diseases. ${ }^{54}$ In fact, apart from hydrotherapy, in the SPA resorts, venous patients may undergo intensive PT and APA programs, diet, and social activities. ${ }^{54}$

A few studies have evaluated the effects of combined therapies performed during thermal cures. These improve QoL as well as venous symptoms. ${ }^{50-54}$ No study has compared the effects of the same protocols performed during thermal cures or in other circumstances.

\section{Psychosocial support}

The relationships between venous disease and psychosocial factors have been evaluated only in subjects afflicted with severe CVI. It has been demonstrated that approximately one-third of patients with $\mathrm{C} 5-\mathrm{C} 6$ disease can be considered "at-risk" of depression. ${ }^{55}$ Moreover, the presence of venous leg ulcers is significantly associated with being single, low self-efficacy levels, ${ }^{55}$ and receiving poorer levels of social support. ${ }^{56}$

Psychosocial measures are significantly associated with a reduced rate of ulcer recurrence. ${ }^{55,56}$ In addition to a direct effect on the immune system and normal healing processes, this can also be due to the impact on self-care and physical activities as well as to better adherence to compression therapy. ${ }^{57,58}$ The strong and statistically significant relationship between social support and recurrence highlights the necessity of increased measures of social interaction in patients with severe CVI. ${ }^{55}$

Measures of psychologic support and social interaction may be necessary in selected patients with CVI, to influence the level of physical activity and self-management, thus allowing a greater adherence to treatments (especially to the use of MECS). ${ }^{57,59}$

\section{Discussion}

This document is the first to present a comprehensive overview of all aspects of rehabilitation for patients with venous diseases of the lower limbs. An exact definition of "venous rehabilitation" is also reported.
Table 4. The "Decalogue" of venous rehabilitation: main diagnostic and therapeutic steps of venous rehabilitation.

\begin{tabular}{ll}
\hline To evaluate & To prescribe \\
\hline - Body mass index & - Proper nutrition, APAs, \\
& sport activity \\
- Plantar load & - Plantar abnormalities correction \\
- Ankle ROM & - Specific PT protocols \\
- Muscle efficiency & - Specific PT protocols \\
- Gait analysis & - Gait re-education \\
- Respiratory dynamics & - Respiratory gymnastic \\
- Comorbidities & - Specific treatment, if possible \\
- Sport activity & - Appropriate sports, elastic \\
& stockings \\
- Sedentarism & - APAs, sports activities \\
- Psychosocial conditions & - Psychic, social, and \\
& occupational supports \\
\hline
\end{tabular}

ROM: range of motion; PT: physical therapy; APAs: adapted physical activities.

The main target of rehabilitation is to prevent disease progression and complications as well as to improve QoL. A holistic approach to venous patients seems indicated, not limited to medical or surgical interventions, but including any form of intervention which may enhance venous return. Venous rehabilitation implies improvement of daily lifestyle and self-care strategies including compression therapy, as well as the adherence to physical rehabilitative protocols.

It is not possible to propose a standard rehabilitative protocol with more details than those in Table 4 . The main reason is that strategies should be individualized according to the severity of the disease, the location and pattern of venous lesion(s), age, motor disabilities, comorbidities, and psychosocial conditions of each patient. The second reason is related to the high costs of the rehabilitative protocols (especially PT, AFA, and SPA) and the marked geographic differences regarding their reimbursement and availability.

Beyond cost implications, the main cause of the poor adherence to rehabilitative protocols is the lack of information from caregivers. ${ }^{44}$ This lack is probably due to the absence of any reference to rehabilitation in studies evaluating the outcome of medical or surgical procedures as well as in most guidelines.

It must be taken into the right account that the majority of studies on rehabilitation of patients with venous diseases of the lower limbs lack scientific evidence. In fact, many investigations demonstrated the efficacy of single rehabilitative tools (e.g. calf exercises) on single components of the venous return (e.g. calf pump) but failed to prove objective benefits in clinical terms (e.g. prevention of ulcer formation and recurrence, reduced rate of recurrent thrombosis, 
or deterioration of PTS). The protocols developed so far need to be discussed and refined by further evidence-based investigations. ${ }^{60}$ In particular, welldesigned randomized controlled trials are required to evaluate the efficacy of the described rehabilitative protocols in influencing the long-term evolution of CVI. On the other hand, it seem desirable that studies evaluating the efficacy of any pharmacologic or surgical treatment consider the prescription and the adherence to rehabilitative protocols, in most cases limited to MECS.

\section{Declaration of Conflicting Interests}

The author(s) declared no potential conflicts of interest with respect to the research, authorship, and/or publication of this article.

\section{Funding}

The author(s) received no financial support for the research, authorship, and/or publication of this article.

\section{Ethical approval}

Not applicable.

\section{Guarantor}

AC.

\section{Contributorship}

AC made a substantial contribution to the concept and design of the work, acquisition of data. AC, MdM, AtC, NM, GM drafted the article and revised it critically for important intellettual content. AC, MdM, AtC, NM, GM approved the version to be published. Each author has participated sufficiently in the work to take public responsability for appropriate portions of the content.

\section{Acknowledgments}

Claudio Allegra, Ludmilla Belova, Patrick Carpentier, Jose Maria Pereira de Godoy, Juergen, Fedor Lurie, Antonio Mander, Hugo Partsch, Adrien Albert Ramelet, Angelo Scuderi, Armando Shapira and Robert Simkin are kindly acknowledged for their contribution to this study.

\section{References}

1. Caggiati A. Highlights of the UIP Consensus on rehabilitation in chronic venous diseases. In: 27th world congress of the IUA, Lyon, 5-8 October 2016.

2. Wittens C, Davies AH, Bækgaard N, et al. Editor's choice - management of chronic venous disease: clinical practice guidelines of the European Society for Vascular Surgery (ESVS). Eur J Vasc Endovasc Surg 2015; 49: 678-737.

3. van Rij AM, De Alwis CS, Jiang P, et al. Obesity and impaired venous function. Eur J Vasc Endovasc Surg 2008; 35: 739-744.
4. Kahn SR, Galanaud JP, Vedantham S, et al. Guidance for the prevention and treatment of the post-thrombotic syndrome. J Thromb Thrombolysis 2016; 41: 144-153.

5. Skervin AL, Thapar A, Franchini AJ, et al. Systematic review and meta-analysis of utility of graduated compression stockings in prevention of post-thrombotic syndrome. Eur J Vasc Endovasc Surg 2016; 51: 838-845.

6. Shingler S, Robertson L, Boghossian S, et al. Compression stockings for the initial treatment of varicose veins in patients without venous ulceration. Cochrane Database Syst Rev 2011; 11: CD008819.

7. Özdemir ÖC, Sevim S, Duygu E, et al. The effects of short-term use of compression stockings on health related quality of life in patients with chronic venous insufficiency. J Phys Ther Sci 2016; 28: 1988-1992.

8. Amsler F, Willenberg T and Blättler W. Management of venous ulcer: a meta analysis of randomized studies comparing bandages to specifically designed stockings. J Vasc Surg 2009; 50: 668-674.

9. Nelson EA and Bell-Syer SE. Compression for preventing recurrence of venous ulcers. Cochrane Database Syst Rev 2014; 9: CD002303.

10. Ramelet AA, Perrin M, Kern P, et al. Phlebology, 5th ed. Issy les Moulineaux: Elsevier-Masson, 2008.

11. Brown A. Life-style advice and self-care strategies for venous leg ulcer patients: what is the evidence? $J$ Wound Care 2012; 21: 342-344, 346, 348-350.

12. Klonizakis M, Tew G, Michaels J, et al. Exercise training improves cutaneous microvascular endothelial function in post-surgical varicose vein patients. Microvasc Res 2009; 78: 67-70.

13. Klonizakis M, Tew G, Michaels J, et al. Effects of upperlimb exercise on lower limb cutaneous microvascular function in post-surgical varicose-vein patients. Eur $J$ Appl Physiol 2010; 109: 1221-1224.

14. Caldwell K, Prior SJ, Kampmann M, et al. Upper body exercise increase lower extremity venous blood flow in deep venous thrombosis. J Vasc Surg Venous and Lym Dis 2013; 1: 126-133.

15. Ludbrook J. The musculovenous pumps of the human lower limb. Am Heart $J$ 1966; 71: 635-641.

16. Gardner AMN and Fox RH. The return of blood to the heart. London: Libbey, 1989.

17. Uhl JF and Gillot C. Anatomy of the veno-muscular pumps of the lower limb. Phlebology 2015; 30: 180-193.

18. Saggini R, Bellomo RG, Iodice P, et al. Venous insufficiency and foot dysmorphism: effectiveness of viscoelastic rehabilitation systems on veno-muscle system of the foot and of the calf. Int J Immunopathol Pharmacol 2009; 22(3 Suppl): 1-8.

19. Uhl JF, Chahim M and Allaert FA. Compression versus inner sole for venous patients with foot static disorders: a prospective trial comparing symptoms and quality of life. Phlebology 2013; 27: 19-30.

20. Dix FP, Brooke R and McCollum CN. Venous disease is associated with an impaired range of ankle movement. Eur J Vasc Endovasc Surg 2003; 25: 556-561.

21. Cavalheri G Jr, de Godoy JM and Belczak CE. Correlation of haemodynamics and ankle mobility with clinical classes of clinical, aetiological, anatomical and 
pathological classification in venous disease. Phlebology 2008; 23: 120-124.

22. Back TL, Padberg FT Jr, Araki CT, et al. Limited range of motion is a significant factor in venous ulceration. J Vasc Surg 1995; 22: 519-523.

23. Pereira de Godoy JM, Braile DM and de Fátima Guerreiro Godoy M. Lymph drainage in patients with joint immobility due to chronic ulcerated lesions. Phlebology 2008; 23: 32-34.

24. Davies JA, Bull RH, Farrelly IJ, et al. A home-based exercise programme improves ankle range of motion in long-term venous ulcer patients. Phlebology 2007; 22: 86-89.

25. Klyscz T, Ritter-Schempp C, Junger $M$, et al. Biomechanical stimulation therapy as physical treatment of arthrogenic venous insufficiency. Hautarzt 1997; 48: 318-322.

26. Ramos-González E, Moreno-Lorenzo C, MataránPeñarrocha GA, et al. Comparative study on the effectiveness of myofascial release manual therapy and physical therapy for venous insufficiency in postmenopausal women. Complement Ther Med 2012; 20: 291-298.

27. Christopoulos D, Nicolaides AN, Cook A, et al. Pathogenesis of venous ulceration in relation to the calf muscle pump function. Surgery 1989; 106: 829-835.

28. Yang D, Vandongen YK and Stacey MC. Changes in calf muscle function in chronic venous disease. Cardiovasc Surg 1999; 7: 451-456.

29. Taheri SA, Heffner R, Pendergast D, et al. Myopathy in venous insufficiency. Phlebology 1987; 2: 7-12.

30. Yim E, Vivas A, Maderal A, et al. Neuropathy and ankle abnormalities in patients with chronic venous disease. JAMA Dermatol 2014; 150: 385-389.

31. Williams KJL, Avekolove O, Moore HM, et al. The calf muscle revisited. J Vasc Surg Venous Lymphat Disord 2014; 2: 329-334.

32. O'Brien J, Edwards H, Stewart I, et al. A home-based progressive resistance exercise programme for patients with venous leg ulcers: a feasibility study. Int Wound $J$ 2013; 10: 389-396.

33. Kahn SR, Shrier I, Shapiro S, et al. Six-month exercise training program to treat post-thrombotic syndrome: a randomized controlled two-centre trial. CMAJ 2011; 183: 37-44.

34. Bogachev VY, Golovanova OV, Kuznetsov AN, et al. Electromuscular stimulation with VEINOPLUS ${ }^{\circledR}$ for the treatment of chronic venous edema. Int Angiol 2011; 30: $567-590$.

35. Izumi M, Ikeuchi M, Mitani $\mathrm{T}$, et al. Prevention of venous stasis in the lower limb by transcutaneous electrical nerve stimulation. Eur J Vasc Endovasc Surg 2010; 39: 642-645.

36. Griffin M, Nicolaides AN, Bond D, et al. The efficacy of a new stimulation technology to increase venous flow and prevent venous stasis. Eur J Vasc Endovasc Surg 2010; 40: 766-771.

37. Clarke Moloney M, Lyons GM, Breen P, et al. Haemodynamic study examining the response of venous blood flow to electrical stimulation of the gastrocnemius muscle in patients with chronic venous disease. Eur $J$ Vasc Endovasc Surg 2006; 31: 300-305.

38. Nelson EA, Mani R, Thomas K, et al. Intermittent pneumatic compression for treating venous leg ulcers. Cochrane Database Syst Rev 2011; 2: CD001899.

39. Kumar S, Samraj K, Nirujogi V, et al. Intermittent pneumatic compression as an adjuvant therapy in venous ulcer disease. J Tissue Viability 2002; 12: 42-44.

40. de Moura RM, Gomes Hde A, da Silva SL, et al. Analysis of the physical and functional parameters of older adults with chronic venous disease. Arch Gerontol Geriatr 2012; 55: 696-701.

41. van Uden CJ, van der Vleuten CJ, Kooloos JG, et al. Gait and calf muscle endurance in patients with chronic venous insufficiency. Clin Rehabil 2005; 19: 339-344.

42. Franklin KJ and Janker R. Respiration and the venae cavae. J Physiol 1936; 86: 254-269.

43. Miller JD, Pegelow DF, Jacques AJ, et al. Skeletal muscle pump versus respiratory muscle pump: modulation of venous return from the locomotor limb in humans. J Physiol 2005; 563: 925-943.

44. Roaldsen KB, Biguet G and Elfving M. Physical activity in patients with venous leg ulcer - between engagement and avoidance. A patient perspective. Clin Rehabil 2011; 25: 275-286.

45. Kelechi TJ, Green A, Dumas B, et al. Online coaching for a lower limb physical activity program for individuals at home with a history of venous ulcers. Home Healthc Nurse 2010; 28: 596-605.

46. Kan YM and Delis KT. Hemodynamic effects of supervised calf muscle exercise in patients with venous leg ulceration: a prospective controlled study. Arch Surg 2001; 136: 1364-1369.

47. Jull A, Parag V, Walker N, et al. The prepare pilot RCT of home-based progressive resistance exercises for venous leg ulcers. J Wound Care 2009; 18: 497-503.

48. Venerando A and Pelliccia A. Pathophysiology of superficial venous circulation in athletes. Phlebologie 1981; 34 : 289-298.

49. Stanek JM. The effectiveness of compression socks for athletic performance and recovery. J Sport Rehabil 2017; 26: 109-114.

50. Petraccia L, Mennuni G, Fontana M, et al. The possible uses of balneotherapy in treating chronic venous insufficiency of lower limbs. Clin Ter 2013; 164: 233-238.

51. Gianesini S, Tessari M, Bacciglieri P, et al. A specifically designed aquatic exercise protocol to reduce chronic lower limb edema. Phlebology 2016; 32: 594-600.

52. Ernst E, Saradeth $\mathrm{T}$ and Resch KL. Hydrotherapy for varicose veins: a randomized, controlled trial. Phlebology 1992; 7: 154-157.

53. Hartmann B, Drews B and Bassenge E. Venous capacity and venous outflow in healthy patients and patients with chronic venous insufficiency before and following swimming in 34 degree C warm water. Vasa Suppl 1991; 32: 443-445.

54. Carpentier PH, Blaise S, Satger B, et al. A multicenter randomized controlled trial evaluating balneotherapy in patients with advanced chronic venous insufficiency. J Vasc Surg 2014; 59: 447-454. 
55. Finlayson K, Edwards H and Courtney M. Relationships between preventive activities, psychosocial factors and recurrence of venous leg ulcers: a prospective study. J Adv Nurs 2011; 67: 2180-2190.

56. Souza Nogueira G, Rodrigues Zanin C, Miyazaki MC, et al. Quality of life of patients with chronic venous ulcers and socio-demographic factors. Wounds 2012; 24: 289-292.

57. Heinen MM, van der Vleuten C, de Rooij MJ, et al. Physical activity and adherence to compression therapy in patients with venous leg ulcers. Arch Dermatol 2007; 143: 1283-1288.
58. Weller CD, Buchbinder $\mathrm{R}$ and Johnston RV. Interventions for helping people adhere to compression treatments for venous leg ulceration. Cochrane Database Syst Rev 2013; 9: CD008378.

59. Mallick R, Lal BK and Daugherty C. Relationship between patient-reported symptoms, limitations in daily activities, and psychological impact in varicose veins. J Vasc Surg Venous Lymphat Disord 2017; 5: 224-237.

60. Araujo DN, Ribeiro CTD, Maciel ACC, et al. Physical exercise for the treatment of non-ulcerated chronic venous insufficiency. Cochrane Database Syst Rev 2016; 12: CDO010637. 